\title{
Neuroimaging in Cerebral Palsy: A Clearer Vision of Neuroplasticity*
}

\section{J. P. Phillips}

Bibliography

DOI $10.1055 / \mathrm{s}-2007-991152$

Neuropediatrics 2007;

38: $112-113$

(c) Georg Thieme Verlag KG

Stuttgart · New York

ISSN 0174-304X

\section{Correspondence}

\section{J. P. Phillips, MD}

The MIND Institute Department of Neurology University of New Mexico Health Science Center 1101 Yale Blvd NE Albuquerque

87131 New Mexico

USA

Tel.: + 1/505/2725028

Fax: + 1/505/272 8002

jpphillips@salud.unm.edu
In the late $1600 \mathrm{~s}$, Anthony Leeuwenhoek of Holland looked at a drop of water with one of his special lenses. What he saw was astonishing. Teeming worlds of life swam before him, exposing for the first time the world of bacteria, protozoa, sperm, and blood cells. This eventually led to a much deeper understanding of disease and treatment, and medicine was never the same again. The scientific paradigm of the day shifted. A new paradigm shift is underway due to recent advances in neuroimaging. Gross anatomy is now clearly defined with conventional MR imaging, and newer techniques go further, demonstrating regional concentrations of neurometabolites using spectroscopic imaging, areas of cortical activation with functional MRI (fMRI), fiber tracking with diffusion tensor imaging (DTI) and temporal processing with magnetoencephalography. Direct cortical stimulation is also possible using transcranial magnetic stimulation (TMS). A particular advantage that any clinician caring for children (or parent, for that matter) will appreciate is that these modalities do not involve radiation or injections. Care must be taken not to over-interpret findings and modifications are often required to effectively scan children, but as non-invasive probes of neuronal function, the potential they offer is tremendous.

This issue of Neuropediatrics includes an important paper by Juenger et al. [5]. 10 children and adults with hemiplegic cerebral palsy were treated with constraint induced movement therapy (CIMT), all of whom demonstrated functional improvement. What sets this paper apart from the burgeoning number of clinical reports on CIMT therapy is that, for this study, subjects were chosen based on meaningful neuroimaging criteria; using TMS and fMRI, all had evidence of late gestation middle cerebral artery infarctions with intact crossed corticospinal and somatosensory projections. Thus, the subjects had similar lesions, occurring at similar gestational ages, with similar neuroanatomic organization. Furthermore, fMRI was improved by controlling subject effort as much as possible during scanning, and by recording the amount of pressure exerted by each subject during each scan. Trials were discarded if the total pressure generated by the subject during scanning was greater after therapy than before. This important modification ensured that changes in cortical activation noted

*Re: Juenger H, Linder-Lucht M, Walther M, Berweck S, Mall V, Staudt, M. Cortical neuromodulation by constraint-induced movement therapy in congenital hemiparesis: An fMRI study on fMRI were not a consequence of greater performance during scanning, but likely due to neuroplastic changes occurring in the brain.

There are myriad causes of cerebral palsy, all of which result in some type of neuronal dysfunction. Treating this dysfunction has been a problem. Until now, it has been difficult to understand functional neuroanatomy in a living patient, much less probe mechanisms of neuroplasticity in response to therapy. With neuroimaging, however, this may be possible. Recent functional imaging studies of children with cerebral palsy have shown that not all subjects with spastic quadriplegia have the same fiber tract involvement [4], that subjects with hemiplegia may have uncoupled motor and sensory projections [13], that neurochemistry is altered at sites distant from visible lesions in children with spastic diplegia [7], and that lesion size determines whether crossed corticospinal projections are present [11]. Clearly, important differences in neurophysiology may underlie similar clinical presentations, and this issue's article by Juenger offers one approach to using neuroimaging as a method of more accurately selecting subjects for clinical investigation.

The real benefit of functional neuroimaging in cerebral palsy will come as future interventional studies include imaging metrics correlated to clinical response. For example, in adults with stroke, DTI may help predict clinical response to upper extremity motor therapy [12], and fMRI may offer information regarding optimal duration of a gait training program [3]. It is possible that some day functional neuroimaging may offer a method of appropriately selecting treatment for specific patients, or that the appropriate duration or intensity of therapy can be determined from imaging data. Novel approaches to therapy may be identified by understanding underlying mechanisms of neuroplasticity, which could include various physical therapies combined with pharmacotherapy [10] or electrical therapy [1].

However, as with any new technology, results currently must be interpreted with caution. Particularly with fMRI, issues such as subject effort $[2,6,8]$, controlling head movement [9], and understanding the effect of growth and medications must be considered. In the example of fMRI, it is not simply a matter of documenting enlargement of cortical activation with therapy, but an issue of determining changes in the extent and location of activation that is important. Probing mechanisms of neuroplasticity such as synaptogenesis, long-term potentiation, cortical reor- 
ganization or neurogenesis will require modification of existing techniques. Colorful pictures are easy to obtain, but it is essential to look beyond the pictures to obtain meaningful information. Like Leeuwenhoek four centuries ago, we are seeing things never seen before. Ultimately this will tremendously impact treatment for children with cerebral palsy. However, much work remains to be done to optimize the use of functional neuroimaging in studying cerebral palsy and other developmental disorders. Clearly, this is work worth pursuing.

\section{Affiliation}

Department of Neurology, University of New Mexico Health Science Center, Albuquerque, New Mexico, USA

\section{References}

1 Boggio PS, Nunes A, Rigonatti SP, Nitsche MA, Pascual-Leone A, Fregni F. Repeated sessions of noninvasive brain DC stimulation is associated with motor function improvement in stroke patients. Restor Neurol Neurosci 2007; 25: 123-129

2 Cramer SC, Weisskoff RM, Schaechter JD, Nelles G, Foley M, Finklestein SP, Rosen $B R$. Motor cortex activation is related to force of squeezing. Human Brain Mapping 2002; 16: 197-205

3 Dobkin BH, Firestine A, West M, Saremi K, Woods R. Ankle dorsiflexion as an fMRI paradigm to assay motor control for walking during rehabilitation. Neuroimage 2004; 23: 370-381
4 Hoon Jr AH, Lawrie Jr WT, Melhem ER et al. Diffusion tensor imaging of periventricular leukomalacia shows affected sensory cortex white matter pathways. Neurology 2002; 59: 752-756

5 Juenger $H$, Linder-Lucht $M$, Walther M, Berweck S, Mall V, Staudt $M$. Cortical modulation by constraint-induced movement therapy in congenital hemiparesis-an fMRI study. Neuropediatrics this issue

6 Kastrup A, Kruger G, Neumann-Haefelin T, Glover G, Moseley ME. Changes of cerebral blood flow, oxygenation, and oxidative metabolism during graded motor activation. Neurolmage 2002; 15: 74-82

7 KulakW, Sobaniec W. Proton magnetic resonance spectroscopy $\left({ }^{1} \mathrm{H}-\right.$ MRS) in children with spastic diplegia. Developmental Medicine and Child Neurology 2004; 46: 287-288

8 Liu JZ, Dai TH, Sahgal V, Brown B, Yue GH. Nonlinear cortical modulation of muscle fatigue; a functional MRI study. Brain Research 2002; 957: 320-329

9 MacIntosh BJ, Mraz R, Baker N, Tam F, Staines WR, Graham SJ. Optimizing the experimental design for ankle dorsiflexion fMRI. Neuroimage 2004; 22: 1619-1627

10 Phillips JP, Devier D, Feeney DM. Rehabilitation pharmacology. Journal of Head Trauma Rehabilitation 2003; 18: 342-356

11 Staudt M, Grodd W, Gerloff C, Erb M, Stitz J, Krageloh-Mann I. Two types of ipsilateral reorganization in congenital hemiparesis a TMS and fMRI study. Brain 2002; 125: 2222-2237

12 Stinear CM, Barber PA, Smale PR, Coxon JP, Fleming MK, Byblow WD. Functional potential in chronic stroke patients depends on corticospinal tract integrity. Brain 2007; 130: 170-180

13 Thickbroom GW, Byrnes ML, Archer SA, Nagarajan L, Mastaglia FL. Differences in sensory and motor cortical organization following brain injury early in life. Ann Neurol 2001; 49: 320-327 